\title{
Strong accretion on a deuterium-burning brown dwarf ${ }^{\star}$
}

\author{
F. Comerón ${ }^{1}$, L. Testi ${ }^{1}$, and A. Natta ${ }^{2}$ \\ 1 ESO, Karl-Schwarzschild-Strasse 2, 85748 Garching bei München, Germany \\ e-mail: fcomeron@eso.org \\ 2 Osservatorio Astrofisico di Arcetri, INAF, Largo E. Fermi 5, 50125 Firenze, Italy
}

Received 8 November 2009 / Accepted 1 July 2010

\begin{abstract}
Context. The accretion processes that accompany the earliest stages of star formation have been shown in recent years to extend to masses well below the substellar limit, and even to masses close to the deuterium-burning limit, suggesting that the features characteristic of the T Tauri phase are also common to brown dwarfs.

Aims. We discuss new observations of GY 11, a young brown dwarf in the embedded $\rho$ Ophiuchi cluster.

Methods. We have obtained for the first time low-resolution, long-slit spectroscopy of GY 11 in the red visible region, using the FORS1 instrument at the VLT. The spectral region includes accretion diagnostic lines such as $\mathrm{H} \alpha$ and the CaII infrared triplet. Results. The visible spectrum allows us to confirm that GY 11 lies well below the hydrogen-burning limit, in agreement with earlier findings based on the near-infrared spectral energy distribution. We obtain an improved derivation of its physical parameters, which suggest that GY 11 is on or near the deuterium-burning phase. We estimate a mass of $30 M_{\text {Jup }}$, a luminosity of $6 \times 10^{-3} L_{\odot}$, and a temperature of $2700 \mathrm{~K}$. We detect strong $\mathrm{H} \alpha$ and CaII triplet emission, and estimate from the latter an accretion rate $\dot{M}_{\text {acc }}=$ $9.5 \times 10^{-10} M_{\odot} \mathrm{yr}^{-1}$, which places GY 11 among the objects with the highest $\dot{M}_{\text {acc }} / M_{*}$ ratios measured thus far in their mass range. This might indicate that accretion in GY 11 is driven by the gravitational instability of its circum(sub-)stellar disk. The intense $\mathrm{H} \alpha$ emission contrasts with the previously reported non-detection of $\mathrm{Pa} \beta$ and $\mathrm{Br} \gamma$ emission, and we discuss possible implications of this on the physical characteristics of the region where hydrogen emission is produced. Using archival near-infrared imaging obtained at different epochs, we prove that the $\mathrm{H}_{2}$ emission previously reported in infrared spectra of GY 11 comes from a chance coincidence with Herbig-Haro knots from the nearby source VLA1623 and not from a molecular outflow driven by GY 11. As a byproduct of our observations, we also obtained a spectrum of the neighboring, embedded low-mass star GY 10, which we classify as M 5.5
\end{abstract}

Key words. stars: formation - stars: pre-main sequence - stars: individual: GY 10 - stars: individual: GY 11

\section{Introduction}

Accretion is recognized as one of the most fundamental features in star formation (Hartmann 1998; Bouvier et al. 2007); however, the precise mechanism through which it operates, the factors determining the accretion rates, the dominant mass transport mechanisms across the circumstellar disk, the role of magnetic fields, or the way it relates to the ejection of mass and angular momentum through disk winds and jets all remain challenging unsolved questions (Hartmann et al. 2006; Ferreira et al. 2006; Shang et al. 2007). Observationally determining of the properties of accreting pre-main sequence stars, by sampling as wide a range as possible of masses, accretion rates, disk properties, viewing geometries, etc. (e.g. Luhman et al. 2005; Mohanty et al. 2005a,b; Damjanov et al. 2007; Evans et al. 2009), is an important task in constraining possible models. In particular, the accumulation of observations of accretion signposts among stars and substellar objects of widely varying masses in the past decade has led to discovery of an approximate proportionality between the accretion rate and the square of the mass of the central object spanning three orders of magnitude in the latter (Muzerolle et al. 2003). While such correlation probably has deep implications on the causes that determine the accretion rate, the precise

* Based on observations carried out with the ESO Very Large Telescope, Cerro Paranal, Chile, under program 70.C-0303; and on material retrieved from the ESO Science Archive. physical mechanisms from which it arises remain difficult to identify (Hartmann et al. 2006).

In this paper we present new results for GY 11, a well known low-mass brown dwarf belonging to the $\rho$ Ophiuchi star-forming region (Wilking et al. 2008). Classification-quality spectroscopy in the visible is presented for the first time for this object, also revealing a positive detection of accretion tracers that allow us to estimate its accretion rate. These results confirm a very lowmass for GY 11, albeit not near the deuterium-burning limit as previously reported, and reveal an unexpectedly high accretion rate.

\section{GY 11}

GY 11 (Greene \& Young 1992), also known as Oph 2320.8-1721 (Rieke \& Rieke 1990), CRBR 25 (Comerón et al. 1993), or ISOOph 033 (Bontemps et al. 2001), was first identified as a brown dwarf candidate by Rieke \& Rieke (1990) in their pioneering near-infrared survey of the $\rho$ Ophiuchi embedded cluster. The substellar nature of GY 11 was suggested by its relatively blue infrared colors and faint magnitude, which indicated a lightly embedded, low-luminosity object superimposed on a region of very high column density of dust. Its first spectrum, in the $2 \mu \mathrm{m}$ region, was obtained by Williams et al. (1995), who reported the tentative detection of $\mathrm{NaI}$ and $\mathrm{CO}$ absorption confirming its low temperature. Higher quality spectra in the same wavelength range allowed Wilking et al. (1999) to produce a spectral 
classification of M 6.5 ( \pm 1.5 spectral subtypes). That spectral classification is in good agreement with Cushing et al. (2000), who used both the $H$ and $K$ bands to derive a type M 6.1, with an uncertainty of \pm 1.6 spectral subclasses. A comparison between the temperature and luminosity and the predictions of evolutionary tracks indicated a mass in the $0.02-0.04 M_{\odot}$ range, in agreement with the early estimates based on near-infrared photometry by Greene \& Young (1992), Comerón et al. (1993), and Strom et al. (1995).

More recently, Testi et al. (2002) have used simultaneous spectroscopy in the $J, H$, and $K$ bands to fit the overall spectral energy distribution between 1 and $2.4 \mu \mathrm{m}$ to the spectra of field dwarfs (Natta et al. 2002) and to synthetic spectra (Allard et al. 2001). The best fit to field dwarf spectra was obtained with a significantly later spectral type than those assigned by previous works, M 8.5. The temperature of the synthetic spectrum yielding the best fit to the observed near-infrared low-resolution spectrum was $T_{\text {eff }}=2400 \mathrm{~K}$, obscured by an extinction $A_{V}=$ $7.0 \mathrm{mag}$. Combining this temperature with the existing infrared photometry, Testi et al. (2002) compared the resulting position in the temperature-luminosity diagram with different sets of evolutionary tracks, concluding that GY 11 may have a mass as low as $0.008-0.012 M_{\odot}\left(8-12 M_{\text {Jup }}\right)$ and an age younger than $1 \mathrm{Myr}$, thus placing it near or even below the deuterium-burning limit. The intrinsic parameters derived by using state-of-the-art premain sequence evolutionary tracks give GY 11 the distinction of being one of the first proposed brown dwarf candidates whose status as such has been confirmed by further observations up to the present.

An infrared excess at long wavelengths in GY 11 has been known since its discovery, as Rieke \& Rieke (1990) already reported its detection at $10 \mu \mathrm{m}$. Comerón et al. (1993) used existing measurements longwards of $2 \mu \mathrm{m}$ to perform a crude fit to the spectral energy distribution that required a considerable excess flux at long wavelengths. This infrared excess was confirmed by Comerón et al. (1998) and Bontemps et al. (2001), in both cases using ISOCAM observations. Testi et al. (2002) and Natta et al. (2002) combined the photometry in all the available ISOCAM bands to obtain a best fit to the spectral energy distribution produced by a face-on flared disk, with a central hole whose radius is 3 times that of the central object. These results were confirmed when using new mid-infrared photometry by Mohanty et al. (2004a), although the new fits hinted at a somewhat higher luminosity, temperature and extinction, hence mass, than those previously estimated by Testi et al. (2002). In this work, a lower limit to the disk mass of $10^{-5}-10^{-6} M_{\odot}$ was derived, depending on the assumed dust mid-IR opacity and surface density profile. A total mass of $\sim 10^{-3} M_{\odot}$ was estimated by Walker et al. (2004).

A relatively massive circum(sub-)stellar disk around such a young low-mass object makes GY 11 an interesting target to search for evidence of accretion. However, the evidence for accretion has been elusive to date, and Natta et al. (2004) used their non-detection of the $\mathrm{Pa} \beta$ and $\mathrm{Br} \gamma$ lines to set an upper limit to the accretion rate of $\log \dot{M}_{\mathrm{acc}}\left(M_{\odot} \mathrm{yr}^{-1}\right)<-10.3$.

\section{New observations}

Although faint and moderately obscured, GY 11 is still accessible to spectroscopic observations in the red visible range using 8 m-class telescopes, as shown by the $R I$ magnitudes measured by Comerón et al. (1998). Observations at those wavelengths can provide a much more accurate spectral classification than is possible in the infrared, thanks to the $\mathrm{TiO}$ and $\mathrm{VO}$ bands whose depths and shapes are sensitive functions of the temperature. Furthermore, observations in that range may be expected to be nearly free of veiling due to circumstellar emission that modifies the spectral energy distribution and fills spectral features in the $2 \mu \mathrm{m}$ region (Luhman \& Rieke 1999), or to the accretion shocks near the photosphere, which produce excess emission mainly at blue-ultraviolet wavelengths. Finally, that region includes important diagnostic lines such as $\mathrm{H} \alpha$ and the CaII triplet, as well as forbidden lines such as [OI], [NII], and [SII], commonly found in emission among young stellar objects.

We present here the first spectrum of GY 11 in the red visible spectral region (6000-10000 ̊), obtained using the FORS1 imager and spectrograph at the ESO Very Large Telescope (VLT) on Cerro Paranal, Chile. The observations were carried out in service mode mode during 5 nights in the period between 26 February and 30 March 2003, in 17 individual exposures of $950 \mathrm{~s}$ each, totalling $4.5 \mathrm{~h}$ of exposure time. A grism covering the spectral region was used with a 1 " 0 -wide slit, yielding a resolution of $\lambda / \Delta \lambda \simeq 260$. All observations used an identical setup. The frames were bias-subtracted and flat-fielded with calibration exposures obtained as a part of the standard calibration plan of the observatory. Spectra were then individually extracted and wavelength-calibrated with He-Ne-Ar lamp exposures obtained generally at the end of the same night. Finally, we stacked together all the extracted individual spectra. Unfortunately, no suitable observations of spectrophotometric standards were obtained with the same setup and close in time (within a few weeks) to our observations. For this reason, the relative flux calibration of our spectra is only approximate. This is, however, irrelevant to the results that we discuss here.

The spectrograph slit was oriented in the north-south direction during our observations. For this reason, our long-slit spectra include also those of GY 10 (=Oph 2320.8-1708), a brighter and more obscured member of the $\rho$ Ophiuchi star-forming region that is located due north of GY 11. We extracted and stacked the spectrum of GY 10 in the same way as for GY 11 as a byproduct of our observations.

We also acquired infrared images of the field surrounding GY 11 in the $L(3.8 \mu \mathrm{m})$ and $M(4.7 \mu \mathrm{m})$ bands using the ISAAC instrument at the VLT in imaging mode. The images were obtained on the nights of 20 to 21 June $2003(L)$ and 30 to 31 May $2003(M)$, with exposure times of $53 \mathrm{~s}$ and $101 \mathrm{~s}$, respectively, and they are shown in Fig. 2. The variable sky transparency during the observations did not allow an absolute photometric calibration of the images, but a comparison between the relative fluxes of GY 11 and GY 10 is still possible. The flux ratio between GY 11 and GY 10 decreases from being over 4 at $L$ to $\sim 2.7$ at $M$, a difference that cannot be attributed to extinction since GY 10 is clearly the more obscured object of the pair, as noted above. The decreasing flux ratio must thus be caused by intrinsic infrared excess in GY 11. Likewise, the flux of Oph 2321.2-1719, the faint source appearing in Fig. 2 to the east of GY 11, decreases from being 35\% of the flux of GY 11 at $L$ to less than that value at $M$, as implied by its non-detection in that band ${ }^{1}$, despite the fact that also Oph 2321.2-1719 is more obscured than GY 11. The lower resolution of the ISOCAM images left the possibility open that the infrared excess might come

1 Early results reported by Rieke \& Rieke (1990) and Comerón et al. (1993) suggest a smaller flux difference at $L$ between both sources. However, the $L$ fluxes given in those studies are highly uncertain, particularly for Oph 2321.2-1719 as noted by Comerón et al. (1993). The flux ratio measurement presented here is far more reliable. 

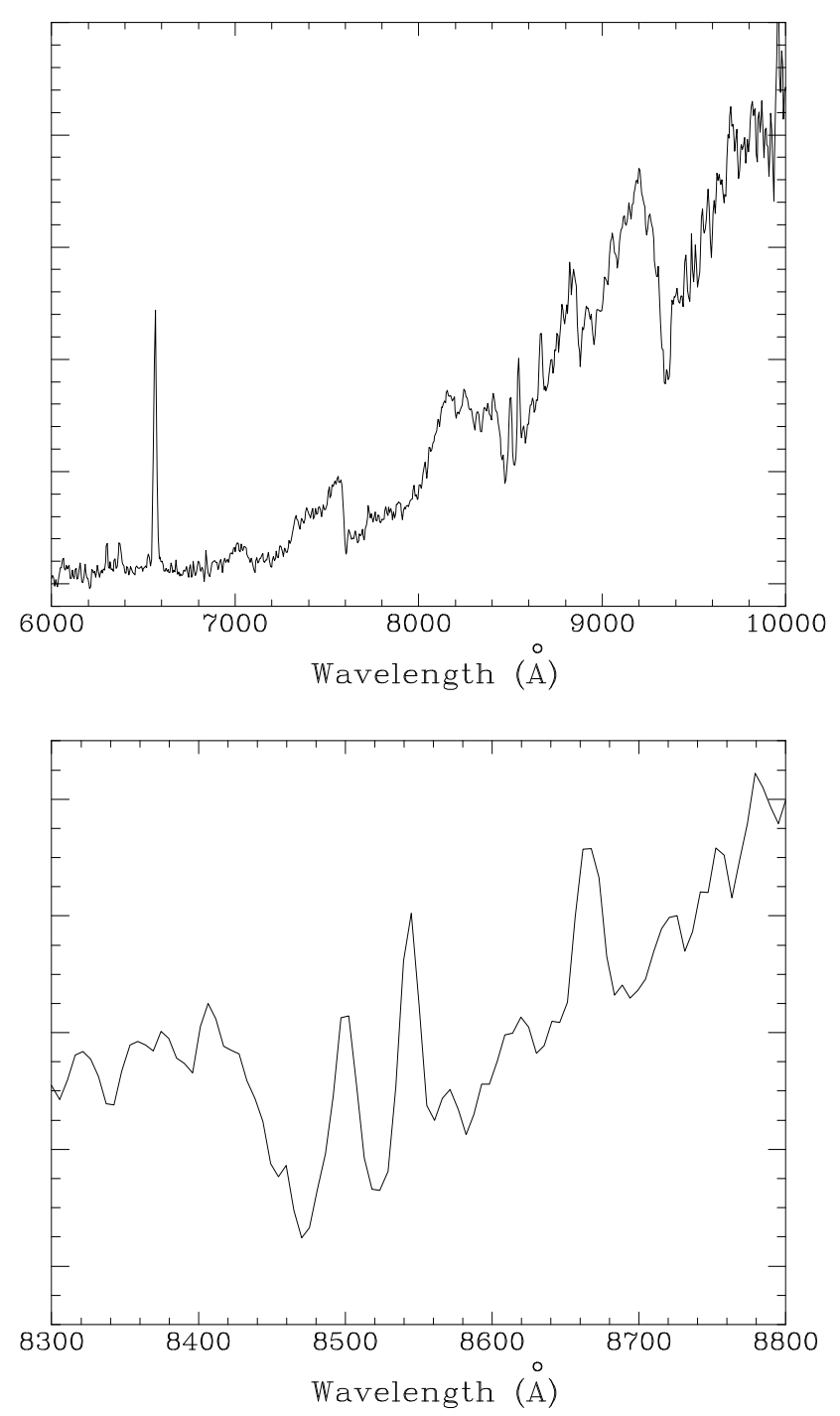

Fig. 1. Visible-red spectrum of GY 11 obtained with FORS1 at the VLT. For clarity, the wavelength region that includes the CaII lines at $8498 \AA$, $8542 \AA$, and $8662 \AA$ is shown as expanded in the bottom panel.

from Oph 2321.2-1719 rather than from GY 11, but the ISAAC results presented here unambiguously settle this issue.

\section{Results}

\subsection{Spectral classification}

The quality of the visible spectrum of GY 11, presented in Fig. 1, is good enough to clarify the discrepancies among the spectral classifications published thus far (Sect. 2), which mainly reflect the limited accuracy with which classification can be carried out in the near infrared. A comparison with the extensive grid of latetype spectra of field stars published by Kirkpatrick et al. (1991) allows us to exclude a spectral type earlier than M 8 mainly based on the shape of the peak between $7300 \AA$ and $7600 \AA$, where a relatively strong VO feature produces a depression blueward of $7500 \AA$. The shape of the TiO bands between $7600 \AA$ and $7800 \AA$, the definite change in slope near $8000 \AA$ and the discontinuity in the pseudocontinuum on both sides of the $\mathrm{TiO}$ feature at $8900 \AA$ are also clear indications of a spectral type later than M 8. On the other hand, the sensitive variation with spectral type of the change of slope at $8000 \AA$, and the height of the pseudocontinuum peak at $8100 \AA$ with respect to the flux just bluewards of the slope break at $8000 \AA$, show that the spectral type must be earlier than M9. We thus classify GY 11 as M 8.5, with an estimated accuracy better than 0.5 spectral subtypes. This agrees with the best fit to the near-infrared spectral energy distribution independently obtained by Testi et al. (2002), which also used a comparison to the spectra of field stars

The low surface gravity of GY 11 is confirmed by the very shallow NaI doublet near $8190 \AA$ (Zhou 1991; Mohanty et al. 2004b); see for instance a comparison with the spectra of field main sequence dwarfs in Kirkpatrick et al. (1991), where this feature is more prominent, and with the sequence of M-type pre-main sequence objects in Comerón et al. (2004) where the $\mathrm{NaI}$ absorption has a similar depth to GY 11.

We are also able to provide an accurate spectral classification for GY 10, whose spectrum is shown in Fig. 3. GY 10 was initially classified as a substantially reddened low-mass star without infrared excess by Comerón et al. (1993) on the basis of its $J H K$ photometry. The fit to the entire spectral energy distribution between 0.6 and $6 \mu \mathrm{m}$ by Comerón et al. (1998) supported this, with an estimated mass of $0.2 M_{\odot}$ and a slight or absent infrared excess between $3.6 \mu \mathrm{m}$ and $6 \mu \mathrm{m}$. However, based on $K$-band spectroscopy, Wilking et al. (1999) assigned an M 8.5 spectral type, apparently indicating a much lower mass. Based on the new visible spectrum we classify GY 10 as M 5.5, also with an uncertainty of \pm 0.5 spectral subtypes, thus closer to earlier estimates that place GY 10 above the hydrogen-burning limit. Its lack of infrared excess and detectable emission lines, including $\mathrm{H} \alpha$, is consistent with an object lacking a warm inner circumstellar disk and significant accretion.

\subsection{The parameters of the central source revisited}

The availability of a spectrum in the visible allows us to determine the temperature of GY 11 by a direct comparison to the grid of synthetic spectra by Allard et al. (2001), which include the low surface gravities expected of young brown dwarfs. The AMES-Dusty models were used, that include dust formation in the atmosphere without gravitational settling and are appropriate for temperatures above $T_{\text {eff }}>1700 \mathrm{~K}$. While a comparison with these models has already been done by Testi et al. (2002) based on the near-infrared spectral energy distribution, the results in that work still left some uncertainty due to remaining problems with the continuum opacities used by the models that affect the near-infrared bands and to some level of degeneracy between the effects of temperature and extinction. Such problems are largely removed by using the red spectral region, where a fair number of molecular features that are highly sensitive to temperature are found, thus leading to a much more reliable estimate of the temperature independently of the extinction and of the fit to the overall spectral energy distribution.

Our results are shown in Fig. 4, where we compare the spectrum of GY 11 with synthetic spectra from Allard et al. (2001) for low-gravity objects $(\log g=3.5)$. The significant changes in spectral features that take place within a narrow temperature range allow us to reliably constrain that of GY 11 to $T_{\text {eff }}=2700 \pm 100 \mathrm{~K}$, in good agreement with the temperature scale vs. spectral type calibration for young low-mass objects of Luhman et al. (2003). The evolutionary models of Chabrier et al. (2000), which incorporate the same set of model atmospheres as a boundary condition, predict an intrinsic $(J-H)_{0}=0.42$ for this temperature, with a very weak dependence on surface 


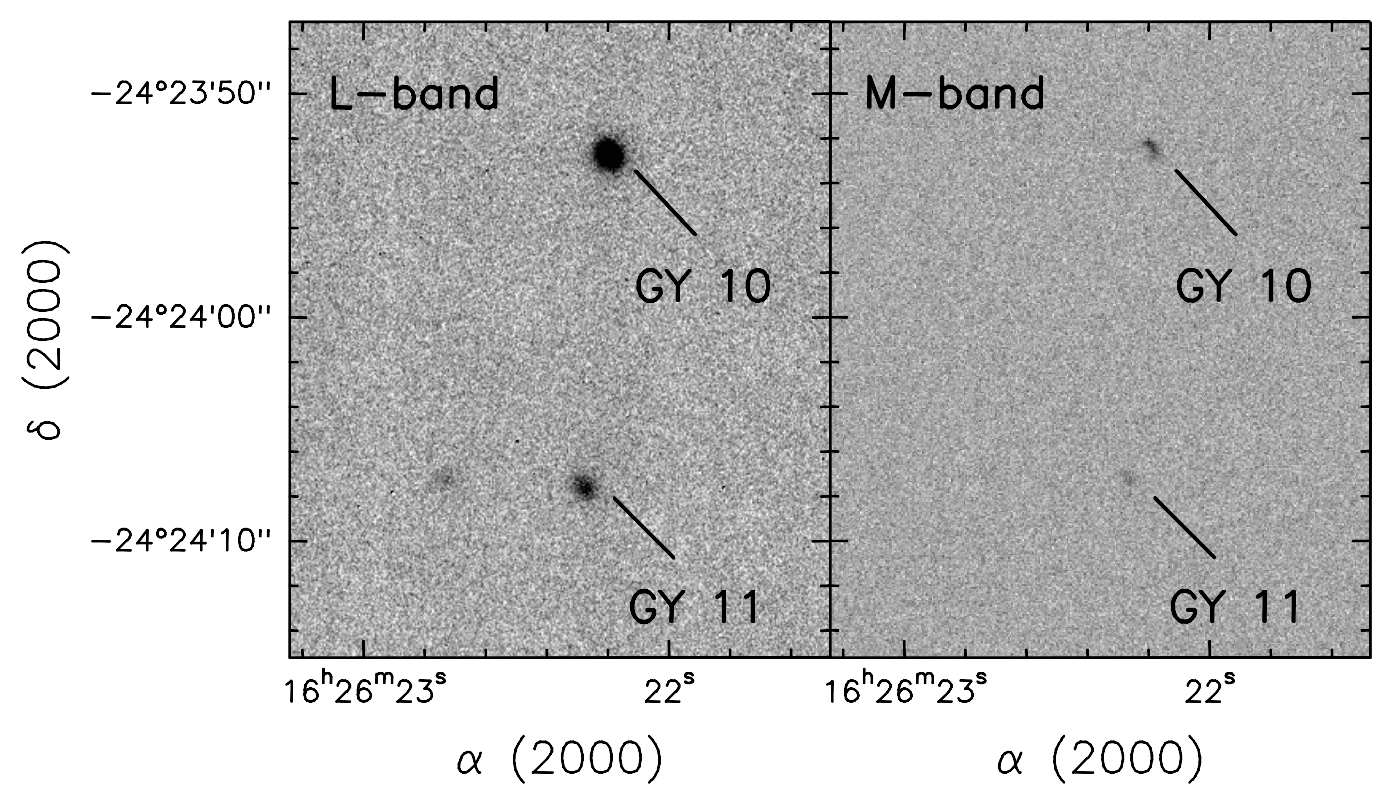

Fig. 2. ISAAC $L$ - and $M$-band images of the region around GY 11 .

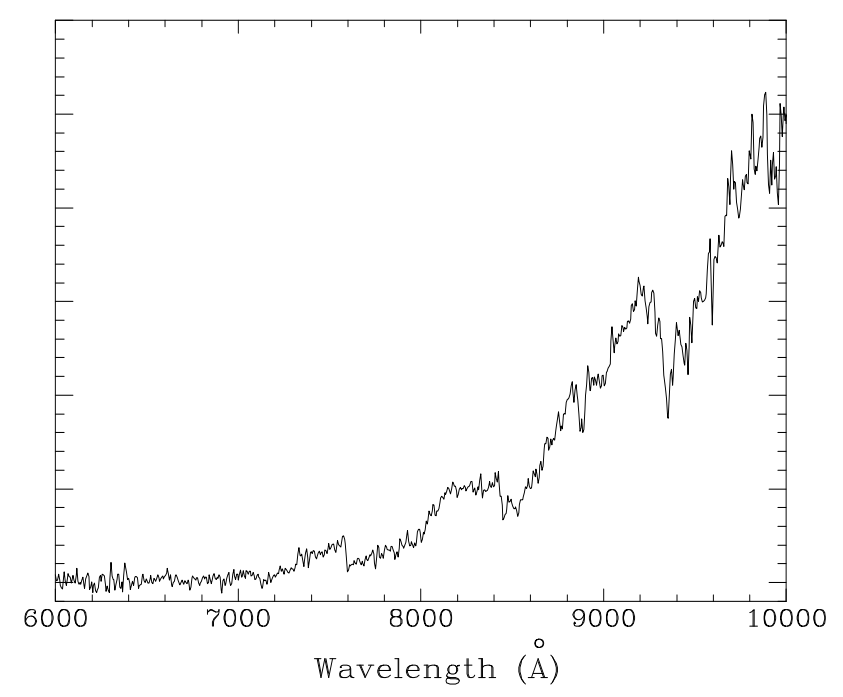

Fig. 3. Spectrum of GY 10, which we classify as M 5.5, obtained simultaneously with the spectrum of GY 11 presented in Fig. 1.

gravity. The 2MASS value $(J-H)=1.36$ confirms a moderate extinction when compared to other members of the $\rho$ Ophiuchi cloud located in the proximities of GY 11. The extinction law in $\rho$ Ophiuchi seems to be represented best by a total-to-selective extinction ratio $R_{V}=5.5$ rather than the characteristic value 3.1 of the diffuse interstellar medium, as discussed by Chapman et al. (2009), which also agrees with previous studies of the region (e.g. Kenyon et al. 1998). We used this value in the parametrization of the extinction law of Cardelli et al. (1989) and used it to fit the near-infrared spectrum obtained by Testi et al. (2002) with a synthetic spectrum at the fixed temperature $T_{\text {eff }}=2700 \mathrm{~K}$, thus removing the coupling between temperature and extinction effects that affected the earlier analysis of Testi et al. (2002). We obtain $A_{J}=2.67$ for GY 11, which corresponds to $A_{V}=8.0$. The latter value is slightly higher than was used in Testi et al. (2002), but closer to the estimate of

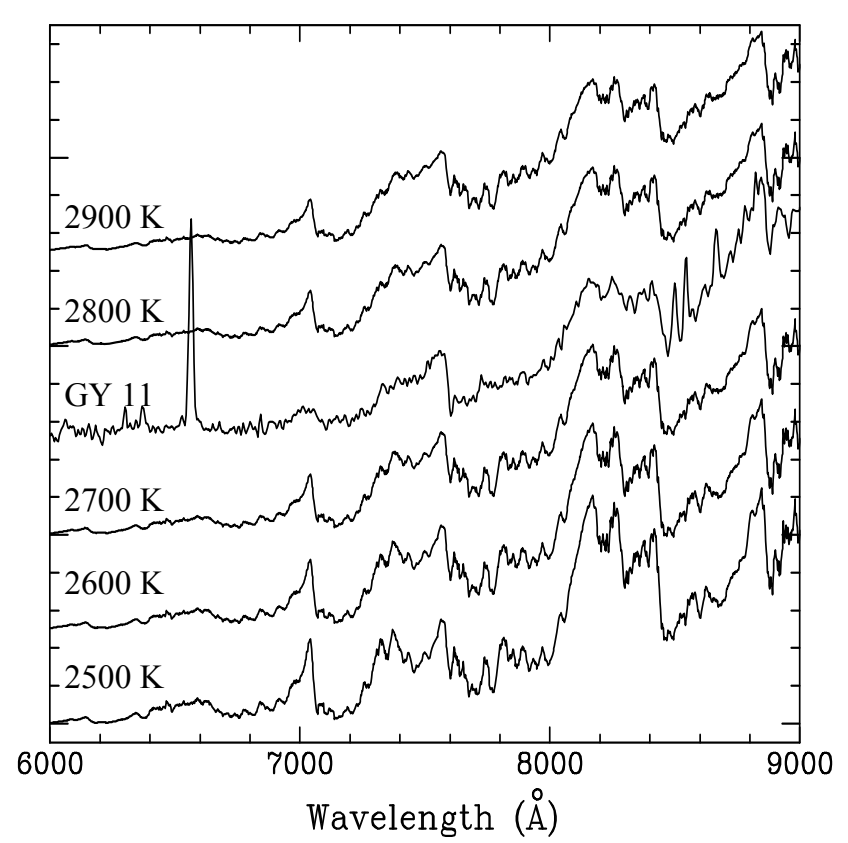

Fig. 4. Comparison of the spectrum of GY 11 with the synthetic spectra of objects with $\log g=3.5$ and different $T_{\text {eff }}$. A spectrum with $T_{\text {eff }}=2700 \mathrm{~K}$ produces the best fit. Given that no spectrophotometric calibration of the GY 11 spectrum was possible with the available data (Sect. 3), the combined effects of reddening and wavelength-dependent instrumental response on the spectrum of GY 11 have been accounted for by computing the ratio of a low-order polynomial fit to it and to the original synthetic spectra. The synthetic spectra have then been multiplied by this ratio, and the results are the spectra displayed in the figure. In this way the overall slopes of the spectra of GY 11 and the comparison spectra are the same, which facilitates their comparison.

Mohanty et al. (2004b) from a fit to the entire spectral energy distribution including mid-infrared data points.

Besides the new determinations of the temperature and extinction discussed above, it is necessary to revise the intrinsic parameters of GY 11 also in light of the strong case made by 


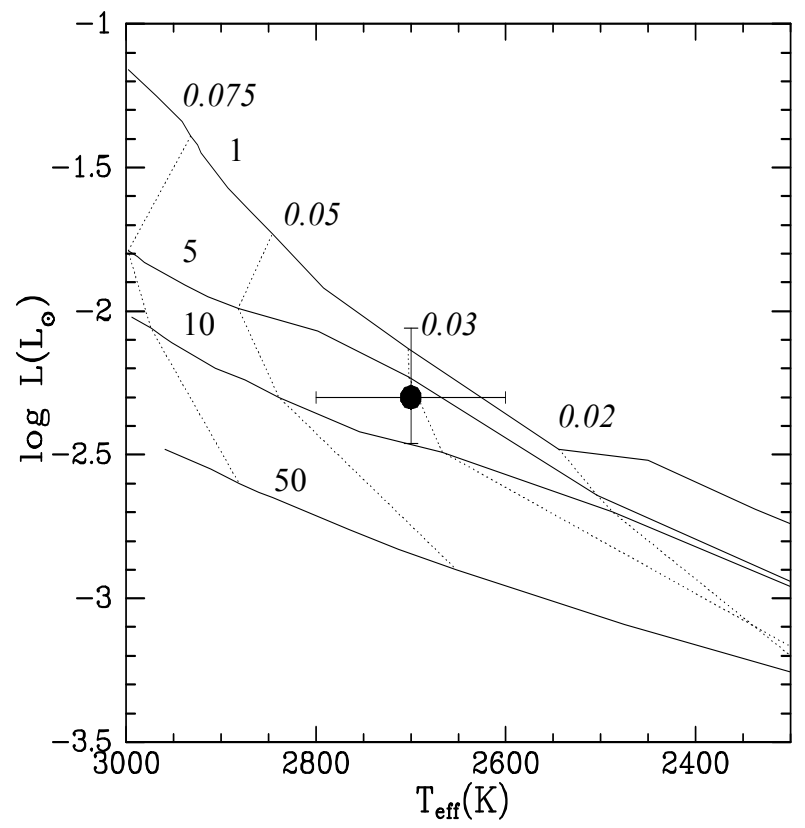

Fig. 5. A comparison between the derived temperature and luminosity of GY 11 and the evolutionary tracks of Chabrier et al. (2000). An uncertainty of $\pm 100 \mathrm{~K}$ in temperature is estimated from comparison to synthetic spectra (Fig. 3). The luminosity is based on the 2MASS $J H K_{\mathrm{S}}$ photometry of GY 11 assuming a distance modulus $D M=5.40$ and a $J$-band extinction $A_{J}=2.67$ obtained from the fit of the infrared spectrum obtained by Testi et al. (2002) to a synthetic spectrum with $T_{\text {eff }}=2700 \mathrm{~K}$ and $\log g=3.5$ (Allard et al. 2001). The ends of the error bar in luminosity account for the different choices of the extinction law and the distance (Sect. 4.2). The model isochrones (solid lines) are labeled in Myr, whereas the numbers in italics indicate the masses corresponding to different evolutionary tracks (dotted lines), in solar masses.

recent studies for a distance to the $\rho$ Ophiuchi cloud less than the value of $160 \mathrm{pc}$ adopted in most previous works; see Wilking et al. (2008) and references therein. In particular, Lombardi et al. (2008) have carried out a robust statistical application of the extinction jump method using the Hipparcos distances to field stars in the direction of $\rho$ Ophiuchi cloud yielding a distance of $119 \pm 6 \mathrm{pc}$, thus reducing the distance modulus $D M$ by $0.6 \mathrm{mag}$ with respect to the widely adopted value and implying a reduction by a factor of almost 2 in the derived luminosities.

Taking as parameters $T_{\mathrm{eff}}=2700 \mathrm{~K}, A_{J}=2.67, D M=5.40$, the 2MASS published value $J=16.45$, and the $J$-band bolometric correction derived from the synthetic spectra of Allard et al. (2001) at the adopted temperature and surface gravity, we obtain a luminosity $\log L\left(L_{\odot}\right)=-2.3$. The position in the temperatureluminosity diagram and its comparison with the evolutionary tracks of Chabrier et al. (2000) are given in Fig. 5, where we also reflect the uncertainties derived from some of the assumptions used. In particular, the lower end of the error bar in luminosity has been calculated by applying the reddening law with $R_{V}=3.1$ instead of $R_{V}=5.5$ while keeping the other parameters unchanged, whereas the upper end is obtained with the "classical" distance modulus $D M=6.0$, again leaving other parameters unchanged.

While our new results firmly suggest a mass near $0.03 M_{\odot}$ in comparison to the evolutionary tracks, the age of GY 11 is very poorly constrained. In fact, our favored choice of parameters as discussed above, indicate that GY 11 is probably located very close to the deuterium-burning main sequence, as one may expect for the vast majority of the objects with a mass similar to that of GY 11 in a star-forming region a few million years old. The temporary stability phase caused by deuterium burning, which lasts up to $\sim 7 \mathrm{Myr}$ at $0.03 M_{\odot}$, is seen in the tight concentration of the isochrones near the position of GY 11, reflecting that the external appearance of the object remains virtually unchanged during this time span.

With the caution required by the significant uncertainties involved in the estimation of intrinsic parameters outlined above and in the evolutionary tracks, the position of GY 11 on or near the deuterium-burning main sequence suggests that evolutionary tracks successfully predict the location of the latter. Furthermore, the relatively strong accretion that GY 11 is undergoing (Sect. 4.3) does not seem to cause any visible modification to its temperature and luminosity, at least while in the deuterium-burning main sequence, despite the possibility that accretion significantly alters the evolutionary paths of low-mass stars and brown dwarfs (Baraffe et al. 2009).

\subsection{Emission lines and accretion rate}

The spectrum of GY 11 shows a prominent $\mathrm{H} \alpha$ line, as well as intense emission in the CaII triplet lines at 8498, 8542, and $8662 \AA$. Possible [OI] emission at 6300 and $6364 \AA$ may be due to residuals from the sky line subtraction at those wavelengths. For the CaII triplet lines we obtained similar equivalent widths of $9.7 \pm 3,8.3 \pm 2$, and $6.6 \pm 0.5 \AA$, respectively, indicating formation in an optically thick medium. For the $\mathrm{H} \alpha$ line we estimate a much more uncertain equivalent width, $(220 \pm 50) \AA$, due to the faintness of the underlying continuum.

The formation of the CaII triplet lines is commonly attributed to accretion shocks very close to the stellar surface (Batalha et al. 1996). A contribution to CaII emission by chromospheric activity is also possible, although its intensity is normally much lower. Not detecting GY 11 in deep X-ray observations with Chandra by Imanishi et al. (2001) indirectly argues against strong chromospheric activity. The general association of CaII triplet emission with accretion in brown dwarfs, low mass, and solar-type pre-main sequence stars is supported by its good observed correlation with other accretion indicators, such as veiling (Batalha et al. 1996) or the existence of broad wings of the hydrogen lines at the $10 \%$ emission level (Muzerolle et al. 1998; Mohanty et al. 2005b). In particular, the suitability of the CaII 8662 line as an accretion rate indicator is confirmed by its strong correlation with the mass loss rate derived from veiling measurements by Herczeg \& Hillenbrand (2008). We will thus assume here that the observed CaII triplet emission also primarily indicates accretion in GY 11.

The relationship between accretion rate and CaII emission derived by Mohanty et al. (2005b) is

$\log \dot{M}_{\mathrm{acc}}=1.06 \log \left(F_{\text {CaII } 8662}\right)-15.40$

where $\dot{M}_{\text {acc }}$ is the accretion rate expressed in $M_{\odot} \mathrm{yr}^{-1}$ and $F_{\text {CaII }} 8662$ the flux emitted in the CaII 8662 line per unit surface of the star or brown dwarf, expressed in erg $\mathrm{cm}^{-2} \mathrm{~s}^{-1}$. After adopting our newly determined temperature for GY $11, T_{\text {eff }}=2700 \mathrm{~K}$, we have obtained the flux radiated by the photosphere per unit wavelength and unit surface at $\lambda=8662 \AA$ from the AMESDusty models of Chabrier et al. (2000) and Allard et al. (2001) at $\log g=3.5$. The flux in the CaII 8662 line radiated per unit surface is then simply the photospheric flux per unit wavelength multiplied by the equivalent width of the CaII 8662 line. In this way we obtain $F_{\text {CaII } 8662}=1.02 \times 10^{6} \mathrm{erg} \mathrm{cm}^{-2} \mathrm{~s}^{-1}$, which 
translates into $\dot{M}_{\text {acc }}=9.5 \times 10^{-10} M_{\odot} \mathrm{yr}^{-1}$. The direct use of synthetic spectra made possible by the new observations, together with the fact that the empirical expression of Mohanty et al. (2005b) given above relates the accretion rate with the flux emitted in the CaII line per unit surface of the object, removes the need to adopt a distance, radius, and extinction towards it.

That CaII should be at all detectable in our spectrum of GY 11 is quite remarkable. A moderate extrapolation of the $\dot{M}_{\text {acc }} \propto M_{*}^{2}$ relationship mentioned in the introduction down to the estimated mass of GY 11 would yield an expected $\dot{M}_{\text {acc }} \sim$ $10^{-11} M_{\odot} \mathrm{yr}^{-1}$, resulting in an equivalent width of only $\sim 0.1 \AA$ for the CaII 8662 line, well below the detection limit of our spectra. The equivalent width that we measure implies a very high accretion rate given the estimated mass of GY 11, well over one order of magnitude above most of the measurements at the lowest masses (Mohanty et al. 2005a) ${ }^{2}$. Herczeg \& Hillenbrand (2008) have shown that the CaII triplet emission occasionally produces a significant overestimate of the accretion rate when compared to that derived from veiling measurements. However, even if the accretion rate that we obtain from the CaII 8662 line in GY 11 were overestimated by as much as in the most extreme cases reported by Herczeg \& Hillenbrand (2008) (about one order of magnitude with respect to the rate derived from ultraviolet veiling), it would still be above the average $\dot{M}_{\text {acc }} \propto M_{*}^{2}$ relationship. CaII emission has been observed in similarly late-type objects, in particular the M9 Taurus brown dwarf KPNO-Tau 12 (Luhman et al. 2003), with an intensity lower than but still comparable to that of our detection in GY 11.

It is interesting to note that the accretion rate that we estimate from the CaII line is over one order of magnitude above the upper limit to the accretion rate derived by Natta et al. $(2004)^{3}$ from their non-detection of the $\mathrm{Pa} \beta$ in the near-infrared spectrum of GY 11. Determination of the accretion rate from line luminosities carried out by Natta et al. (2004) depends strongly on the assumed value of the extinction. Natta et al. (2004) adopted $A_{V}=7.0 \pm 1.0 \mathrm{mag}$. To reconcile the Natta et al. (2004) upper limit with our estimate of the accretion rate, the extinction should be $A_{V} \simeq 12$ or more, which would be inconsistent with the observations, even when taking the somewhat higher value of $A_{V}$ that we find in the present study. The scatter in the calibrations of mass accretion rates in terms of either $\mathrm{Pa} \beta$ and $\mathrm{CaII}$ triplet emission may account for the disagreement between both estimates. Intrinsic variability of the accretion is another plausible cause.

Another puzzling feature is that the strength of the $\mathrm{H} \alpha$ emission combined with not detecting $\mathrm{Pa} \beta$ appears to contrast with recent results on the formation of hydrogen lines in the magnetospheric accretion shocks of very low-mass stars.

\footnotetext{
${ }^{2}$ Natta et al. (2004) have used infrared hydrogen lines to derive comparable accretion rates, near $10^{-9} M_{\odot} \mathrm{yr}^{-1}$, for other objects in $\rho$ Ophiuchi with estimated masses of $0.04 M_{\odot}$. However, the temperatures and spectral types given in that work are inconsistent with the calibration of Luhman et al. (2003), as in the case of GY 11 discussed in Sect. 4.2. It is thus possible that the masses derived by Natta et al. (2004) may have to be revised upwards, in a similar way to the revised mass estimate of GY 11 presented in this paper.

${ }^{3}$ Natta et al. (2006) give a much lower value than Natta et al. (2004) for the accretion rate. The estimate in the former work is based, for consistency with the rest of the analysis presented in that paper, on a statistical determination of the photospheric parameters for GY11 which resulted in a much lower estimate of the extinction and luminosity. As noted in Natta et al. (2006), for GY11 the statistical method is not as accurate as the detailed analysis, and the value in Natta et al. (2004) is to be preferred instead.
}

Gatti et al. (2006) have investigated the generally small $\mathrm{Pa} \beta / \mathrm{Br} \gamma$ ratios among very low-mass stars with low accretion rates, which are consistent with optically thick emission from a relatively cold $\left(T_{\text {eff }} \sim 3500 \mathrm{~K}\right)$ plasma in local thermodynamic equilibrium (LTE). In an optically thin plasma, Case B recombination theory predicts an intensity ratio $\mathrm{H} \alpha / \mathrm{Pa} \beta=17.5$ at the electron temperature $T_{\mathrm{e}}=10^{4} \mathrm{~K}$, with a very mild dependence on temperature (Pengelly 1964). Using an estimated equivalent width $E W(\mathrm{H} \alpha)=220 \AA$, an upper detection threshold $E W(\mathrm{~Pa} \beta)=$ $0.7 \AA$, and the spectral energy distribution of a young brown dwarf photosphere of $\log g=3.5, T_{\text {eff }}=2700 \mathrm{~K}$ (which gives a photospheric flux ratio at the respective wavelengths of $\mathrm{H} \alpha$ and $\mathrm{Pa} \beta$ of $F_{6563} / F_{122822}=0.13$; Allard et al. 2001), we obtain for GY 11 an intrinsic intensity ratio $\mathrm{H} \alpha / \mathrm{Pa} \beta>40$, which is much higher than the ratio expected from recombination theory. Such a lower limit definitely rules out the lower ratio arising from the formation of both lines in a common optically thick medium. Our results are nevertheless consistent with the high $\mathrm{H} \alpha / \mathrm{Pa} \beta$ ratios expected in an optically thin plasma with an LTE population of excited levels and are in this respect closer to the model predictions of Muzerolle et al. (2001), where the ratio increases as the higher excitation lines, and then the lower excitation ones, become progressively optically thin with decreasing accretion rates. Accretion in GY 11 might probe a region of the parameter space where emission in both $\mathrm{H} \alpha$ and $\mathrm{Pa} \beta$ is dominated by a pre-shock medium that is optically thin thanks to the low accretion rate, while emission from that component weakens at even lower accretion rates and the post-shock hot spots formed in optically thick gas gain prominence in comparison, thus giving validity to the scenario outlined by Gatti et al. (2006). None of the objects examined by those authors appears to be in the regime in which the emission is dominated by optically thin preshock gas with high $\mathrm{Pa} \beta / \mathrm{Br} \gamma$ ratios, which may cast doubt on whether that regime, where we tentatively place GY 11, is ever reached in reality. However, in addition to GY 11, at least four of the very low-mass stars and brown dwarfs in Chamaeleon I and $\rho$ Ophiuchi examined by Natta et al. (2004) have $\mathrm{H} \alpha$ equivalent widths and non-detections in $\mathrm{Pa} \beta$ emission that imply ratios $\mathrm{H} \alpha / \mathrm{Pa} \beta>10$. Of course, we cannot rule out the possibility that our result arguing for a high $\mathrm{H} \alpha / \mathrm{Pa} \beta$ ratio accidentally arises from line variability, since the $\mathrm{Pa} \beta$ observations and our $\mathrm{H} \alpha$ observations are not simultaneous. Although the variability should be rather large in order to produce as marked a deviation from the optically thin recombination ratios as those observed in the $\mathrm{Pa} \beta / \mathrm{Br} \gamma$ ratios, this possibility definitely needs to be investigated with further observations simultaneously covering the $\mathrm{H} \alpha$ and $\mathrm{Pa} \beta$ ranges.

\subsection{Nearby $\mathrm{H}_{2}$ emission}

Despite the strong accretion rates inferred from the CaII triplet lines and the intense $\mathrm{H} \alpha$ emission, the spectrum of GY 11 does not display evidence of the forbidden lines that normally trace jets and other forms of outflows, in which mass is lost by the system at a rate that is roughly $1-10 \%$ of the accretion rate onto the central object (Hartigan et al. 1995). The [OI] lines at 6300 and $6364 \AA$ might be present, but they appear in a zone of the spectrum that is heavily contaminated by telluric emission at those same wavelengths, and their apparent presence in the spectrum shown in Fig. 1 may just be an artifact from the removal of telluric emission at the time of extracting the spectra. More significant is the definite absence of the [SII] lines at 6716 

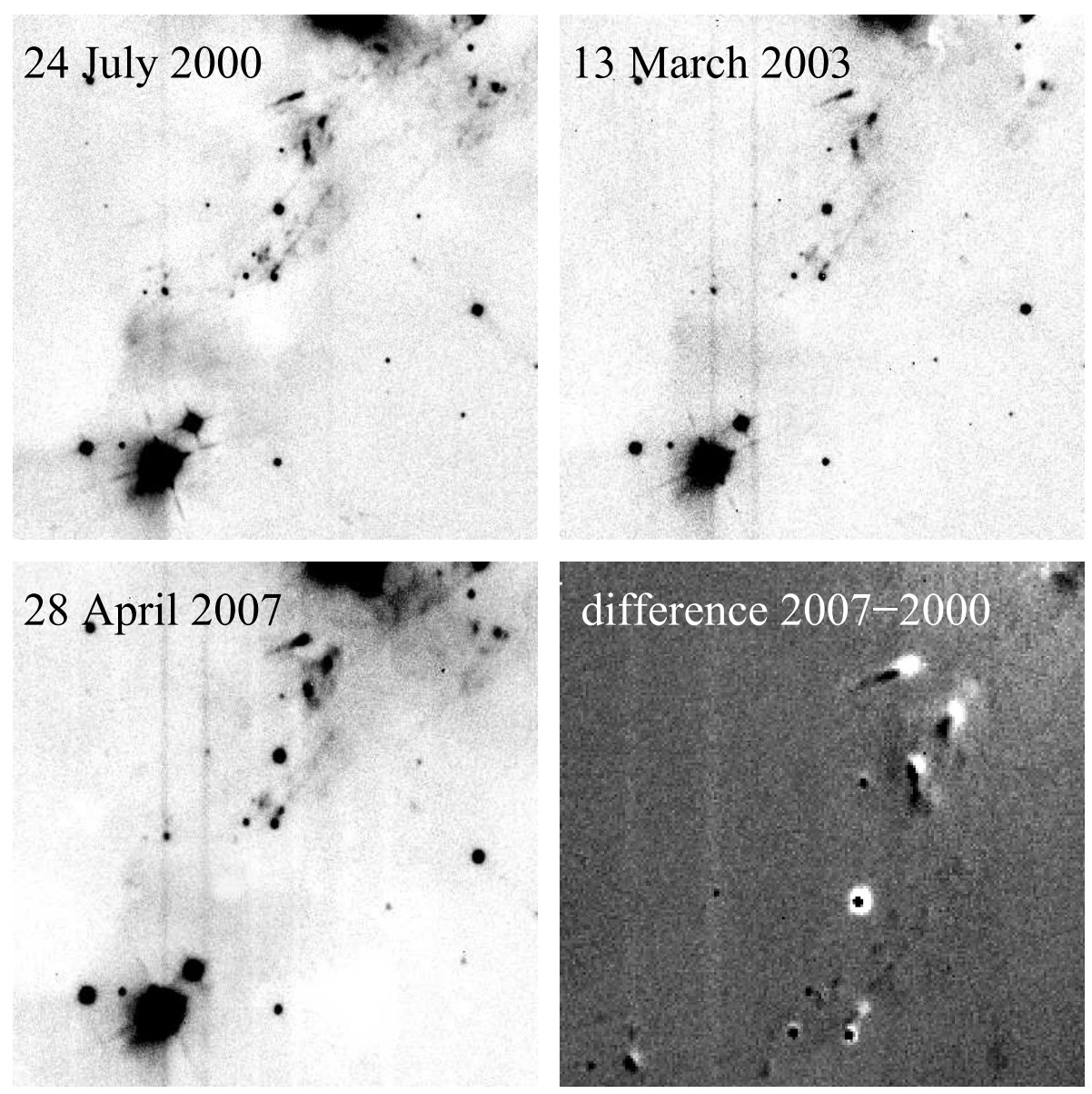

Fig. 6. Archive images obtained with SOFI at the NTT at different epochs of the field around GY 11, which is located at the center of the images in the upper panels and the lower-left panel. The images, taken through a narrowband filter centered at the $2.12 \mu \mathrm{m}$ wavelength of $\mathrm{H}_{2}$, show some other low-mass members of the $\rho$ Ophiuchi cluster in the same area as well as the system of Herbig-Haro $(\mathrm{HH})$ objects having their origin in the VLA1623 source, outside the field to the lower right. The fields of the first three panels are 115" across. The field of the difference image at the bottom right is $58^{\prime \prime}$ across. North is to the top and east to the left.

and $6731 \AA$, down to a flux level that we estimate to be $<2 \%$ that of the $\mathrm{H} \alpha$ line.

The lack of forbidden lines in the spectrum of GY 11 suggests that the detection of extended $\mathrm{H}_{2}$ emission at $2.12 \mu \mathrm{m}$ by Williams et al. (1995), also seen in the spectra of Wilking et al. (1999), may come from a chance alignment with an unrelated Herbig-Haro (HH) knot at the time of those observations. This has been already suggested by Natta et al. (2004), and we have been able to directly prove it by examining archive images of the region where the proper motion of $\mathrm{H}_{2}$ knots, including one very close to the position of GY 11, can be easily measured. The images, presented in Fig. 6, were obtained using the SOFI nearinfrared camera and spectrograph at the ESO New Technology Telescope (NTT) in La Silla, Chile, using a narrow-band filter centered at the wavelength of the $(1,0) \mathrm{H}_{2} S$ (1) line. The observations had been obtained for different programs on the nights of 24 July 2000, 13 March 2003, and 28 April 2007. After identifying them in the ESO Science Archive Facility, we requested them with their corresponding calibration frames and reduced them.

Although the images have differences in depth and in image quality, identifying rapidly moving $\mathrm{HH}$ knots in them is straightforward, as a careful comparison of the images taken on different dates shows. The differences are most clearly seen by subtracting pairs of images obtained at different epochs, as illustrated by the bottom right panel of Fig. 6. In it, stars appear as black dots surrounded by a small white halo owing to the better quality of the images taken in July 2000 as compared to those of April 2007, whereas HH knots have all been displaced by a similar amount to the northwest (upper right in the figure). The proper motion of the knots is found to be $0^{\prime \prime} 31 \mathrm{yr}^{-1}$ with respect to the stars in the field, corresponding to a tangential velocity of $174 \mathrm{~km} \mathrm{~s}^{-1}$ at the distance of 119 pc (Lombardi et al. 2008) directed to a position angle of $315^{\circ}$. Their motion is thus directed radially away from VLA1623, a protostellar object with powerful outflow activity (Motte et al. 1998). The total field of our frames (not shown in Fig. 6) also shows the stream moving southeastwards on the opposite side of VLA1623.

As Fig. 6 shows, one of the HH knots is very close to the position of GY 11 and is moving in the same general direction and with the same velocity as the other knots away from VLA1623, thus showing its clear association with that source. Tracing its path back in time we find that the knot was closest to GY 11 in 1984, when it was located $1^{\prime \prime} 0$ from GY 11 . The knot would have fallen within a $1^{\prime \prime}$ spectrograph slit centered on GY 11 and oriented in the north-south direction between 1987 and 1998, less than $2^{\prime \prime}$ away from it, thus contaminating the spectra obtained by both Williams et al. (1995) and Wilking et al. (1999). We can thus definitely rule out the physical association of $\mathrm{H}_{2}$ with GY 11, thus confirming the suggestion by Natta et al. (2004) of a chance alignment.

\section{Discussion and conclusions}

If the CaII triplet emission detected in the spectrum of GY 11 indeed provides a measurement of the accretion rate as indicated by previous work (Sect. 4.3), the very low mass confirmed with our new results implies a very high $\dot{M}_{\text {acc }} / M_{*}$. Late M-type members of clusters discovered in recent years that also 
show hints of accretion in the form of strong $\mathrm{H} \alpha$ emission are generally located in clusters that are somewhat more evolved than $\rho$ Ophiuchi, such as the brown dwarfs in Taurus-Auriga (1-3 Myr; Luhman et al. 2003; Barrado y Navascués 2004; Luhman 2004), the $\lambda$ Ori cluster (5 Myr; Barrado y Navascués et al. 2004), and other nearby clusters (Jayawardhana et al. 2003). However, the location of GY 11 near the deuteriumburning main sequence is consistent with an age in that same range. Dissipation of disks on timescales of several Myr is reflected in the slowly declining fraction of brown dwarfs with disks among clusters of increasing ages (Jayawardhana et al. 2003). In this regard we note that the M9 object KPNO 12 in Taurus, for which Luhman et al. (2003) have reported CaII triplet emission at a level lower than in GY 11, may be an object with very similar characteristics to those of GY 11 seen at a later evolutionary stage.

The remarkably high $\dot{M}_{\text {acc }} / M_{*}$ ratio of GY 11 , and perhaps other brown dwarfs with somewhat higher masses in their initial stages (Muzerolle et al. 2003; White \& Basri 2003; Natta et al. 2004), may have some important implications for the mechanisms driving accretion and the evolution of disks around such masses. The accretion rate that we estimate in Sect. 4.3 places GY 11 close to the upper envelope $\dot{M}_{\text {acc }}=10^{-7} \mathrm{yr}^{-1} M_{*}$ of the $\dot{M}_{\text {acc }} \propto M_{*}^{2}$ relationship, proposed by Hartmann et al. (2006) as the limit at which the initial disk is gravitationally unstable, under the assumption that such an accretion rate can be sustained over at least $\sim 1 \mathrm{Myr}$, implying a disk with a mass $M_{\text {disk }} \sim 0.1 M_{*}$. For GY 11, this agrees with the rough estimate of $M_{\text {disk }} \sim 10^{-3} M_{\odot}$ by Walker et al. (2004), with the caveats entailed by the strong model-dependence of disk mass estimates (D'Alessio et al. 2001). Several stars and brown dwarfs with masses between solar and $0.04 M_{\odot}$ are known to lie close to the $\dot{M}_{\mathrm{acc}}=10^{-7} \mathrm{yr}^{-1} M_{*}$ relationship (Muzerolle et al. 2005), but GY 11 is the lowest mass object known thus far for which disk gravitational instability appears to be the favored accretiondriving mechanism. It may be noted as well that objects near the upper envelope of the $\dot{M}_{\text {acc }}-M_{*}^{2}$ relation are likely to have layered accretion and non-accreting, turbulent regions near the disk midplane (dead zones) that may be the preferred place of formation of planetary companions, suggesting that even isolated brown dwarfs might have their own planetary systems.

The new observations presented in this paper, which include the first spectrum of GY 11 in the visible, directly and accurately confirm its spectral type and are the best way to determine its intrinsic parameters, which indicate that GY 11 is a young brown dwarf on or near the deuterium-burning main sequence. The signatures of relatively strong accretion discovered in the visible spectrum, $\mathrm{H} \alpha$ and CaII triplet emission, together with the already known infrared emission produced by circumstellar dust, fit in the general picture of a brown dwarf possessing a circumstellar environment that reproduces the key features seen among the higher-mass classical T Tauri stars on a smaller scale, adding to the mounting evidence of an essentially single scenario for the formation of solar-type stars, low-mass stars, brown dwarfs, and probably even freely-floating objects of giant planet masses.

Acknowledgements. We are pleased to thank the staff of Paranal Observatory Science Operations for the execution of this program in service mode at the VLT, and to the User Support Department of the ESO Data Management and Operations Division for their careful revision of the Phase 2 package defining our observations. The very helpful comments made by the referee, Subhanjoy Mohanty, led to substantial improvements in this paper.

\section{References}

Allard, F., Hauschildt, P. H., Alexander, D. R., Tamanai, A., \& Schweitzer, A. 2001, ApJ, 556, 357

Baraffe, I., Chabrier, G., \& Gallardo, J. 2009, ApJ, 702, L27

Barrado y Navascués, D. 2004, A\&A, 422, 631

Barrado y Navascués, D., Stauffer, J. R., Bouvier, J., Jayawardhana, R., \& Cuillandre, J.-C. 2004, ApJ, 610, 1064

Batalha, C. C., Stout-Batalha, N. M., Basri, G., \& Terra, M. A. O. 1996, ApJS, 103,211

Bontemps, S., André, P, Kaas, A. A., et al. 2001, A\&A, 372, 173

Bouvier, J., Alencar, S. H. P., Harries, T. J., Johns-Krull, C. M., \& Romanova, M. M. 2007, in Protostars and Planets V, ed. B. Reipurth, D. Jewitt, \& K. Keil (Univ. of Arizona Press)

Cardelli, J. A., Clayton, G. C., \& Mathis, J. S. 1989, ApJ, 345, 245

Chabrier, G., Baraffe, I., Allard, F., \& Hauschildt, P. 2000, ApJ, 542, 464

Chapman, N. L., Mundy, L. G., Lai, S.-P., \& Evans, N. J. 2009, ApJ, 690, 496

Comerón, F., Rieke, G. H., Burrows, A., \& Rieke, M. J. 1993, ApJ, 416, 185

Comerón, F., Rieke, G. H., Claes, P., Torra, J., \& Laureijs, R. J. 1998, A\&A, 335, 522

Comerón, F., Fernández, M., Baraffe, I., Neuhäuser, R., \& Kaas, A. A. 2003, A\&A, 406, 1001

Comerón, F., Reipurth, B., Henry, A., \& Fernández, M. 2004, A\&A, 417, 583

Cushing, M. C., Tokunaga, A. T., \& Kobayashi, N. 2000, AJ, 119, 3019

D'Alessio, P., Calvet, N., \& Hartmann, L. 2001, ApJ, 553, 321

Damjanov, I., Jayawardhana, R., Scholz, A., et al. 2007, ApJ, 670, 1337

Evans, N. J., Dunham, M. M., Jørgensen, J. K., et al. 2009, ApJS, 181, 321

Ferreira, J., Dougados, C., \& Cabrit, S. 2006, A\&A, 456, 785

Gatti, T., Testi, L., Natta, A., Randich, S., \& Muzerolle, J. 2006, A\&A, 460, 547 Greene, T. P., \& Young, E. T. 1992, ApJ, 395, 516

Hartigan, P., Edwards, S., \& Ghandour, L. 1995, ApJ, 452, 736

Hartmann, L. 1998, Accretion Processes in Star Formation (Cambridge Univ. Press)

Hartmann, L., D'Alessio, P., Calvet, N., \& Muzerolle, J. 2006, ApJ, 648, 484

Herczeg, G. J., \& Hillenbrand, L. A. 2008, ApJ, 681, 594

Imanishi, K., Tsujimoto, M., \& Koyama, K. 2001, ApJ, 563, 361

Jayawardhana, R., Ardila, D. R., Stelzer, B., \& Haisch, K. R. 2003, AJ, 126, 1515

Kirkpatrick, J. D., Henry, T. J., \& McCarthy, D. W. 1991, ApJS, 77, 417

Kenyon, S. J., Lada, E. A., \& Barsony, M. 1998, AJ, 115, 252

Lombardi, M., Lada, C. J., \& Alves, J. 2008, A\&A, 480, 785

Luhman, K. L. 2004, ApJ, 617, 1216

Luhman, K. L., \& Rieke, G. H. 1999, ApJ, 525, 440

Luhman, K. L., Stauffer, J. R., Muench, A. A., et al. 2003, ApJ, 593, 1093

Luhman, K. L., D’Alessio, P., Calvet, N., et al. 2005, ApJ, 620, L51

Luhman, K. L., Joergens, V., Lada, C., et al. 2007, in Protostars and Planets V, ed. B. Reipurth, D. Jewitt, \& K. Keil (Univ. of Arizona Press)

Mohanty, S., Jayawardhana, R., Natta, A., et al. 2004a, ApJ, 609, L33

Mohanty, S., Basri, G., Jayawardhana, R., et al. 2004b, ApJ, 609, 854

Mohanty, S., Jayawardhana, R., \& Basri, G. 2005a, ApJ, 626, 498

Mohanty, S., Basri, G., \& Jayawardhana, R. 2005b, Astron. Nachr., 326, 891

Motte, F., André, P., \& Neri, R. 1998, A\&A, 336, 150

Muzerolle, J., Hartmann, L., \& Calvet, N. 1998, AJ, 116, 455

Muzerolle, J., Calvet, N., \& Hartmann, L. 2001, ApJ, 550, 944

Muzerolle, J., Hillenbrand, L., Calvet, N., Briceño, C., \& Hartmann, L. 2003, ApJ, 592, 266

Muzerolle, J., Luhman, K. L., Briceño, C., Hartmann, L., \& Calvet, N. 2005, ApJ, 625, 906

Natta, A., Testi, L., Comerón, F., et al. 2002, A\&A, 393, 597

Natta, A., Testi, L., Muzerolle, J., et al. 2004, A\&A, 424, 603

Natta, A., Testi, L., \& Randich, S. 2006, A\&A, 452, 245

Pengelly, R. M. 1964, MNRAS, 127, 145

Rieke, G. H., \& Rieke, M. J. 1990, ApJ, 362, L21

Shang, H., Li, Z.-Y., \& Hirano, N. 2007, in Protostars and Planets V, ed. B. Reipurth, D. Jewitt, \& K. Keil (Univ. of Arizona Press)

Strom, K. M., Kepner, J., \& Strom, S. E. 1995, ApJ, 438, 813

Testi, L., Natta, A., Oliva, E., et al. 2002, ApJ, 571, L155

Walker, C., Wood, K., Lada, C. J., et al. 2004, MNRAS, 351, 607

White, R. J., \& Basri, G. 2003, ApJ, 5821109

Wilking, B. A., Greene, T. P., \& Meyer, M. R. 1999, AJ, 117, 469

Wilking, B. A., Gagné, M., \& Allen, L. E. 2008, in Handbook of Star Forming Regions, ed. B. Reipurth, ASP Monographs

Williams, D. M., Comerón, F., Rieke, G. H., \& Rieke, M. J. 1995, ApJ, 454, 144 Zhou, X. 1991, A\&A, 248, 367 